\title{
Practice and research
}

\section{Non-invasive detection of hypoglycaemia using a novel, fully biocompatible and patient friendly alarm system}

Mimi Chen, Mark Daly, Natt, Susie, Candy, Gareth Williams

Hypoglycaemia is a common complication of diabetes treatment with either insulin or sulphonylureas and is particularly common with intensified insulin regimens. ${ }^{1}$ Episodes are often distressing and carry the risk of serious neurological and cardiovascular sequelae. Hypoglycaemia is especially hazardous in patients with longstanding type 1 diabetes who have lost the early warning symptoms of falling blood glucose levels. Nocturnal episodes are also potentially dangerous and have been implicated when diabetic patients have been found unexpectedly dead in bed. ${ }^{2}$ Hypoglycaemia is one of the complications of diabetes most feared by patients, on a par with blindness and renal failure. ${ }^{3}$

Intensive research has been devoted to the development of hypoglycaemia alarms, exploiting principles that range from detecting changes in the electroencephalogram or skin conductance (due to
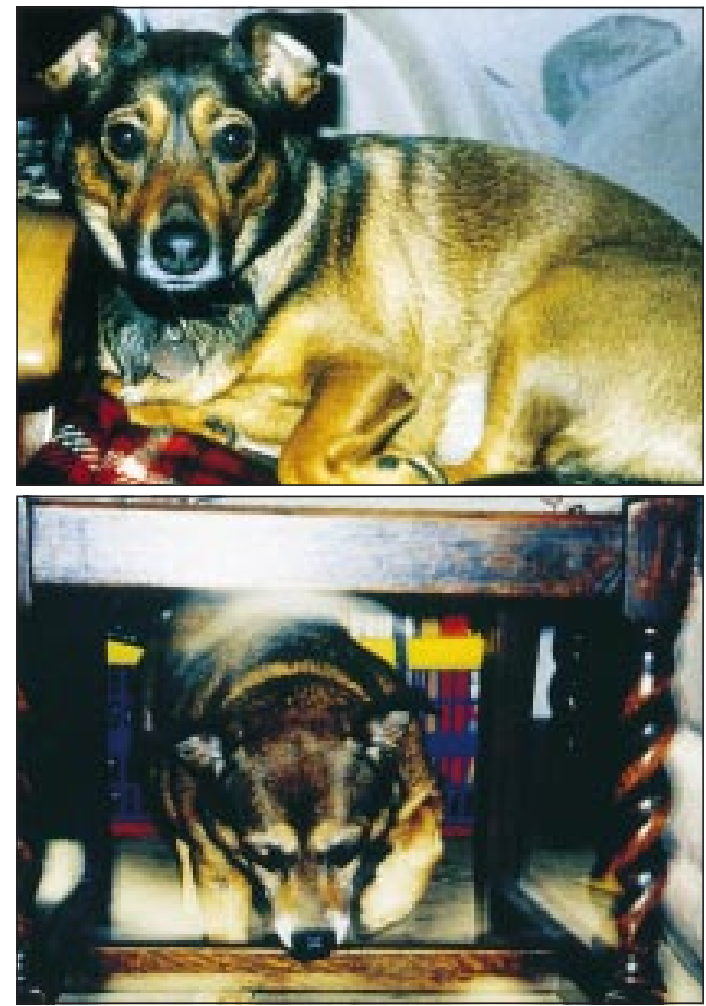

Fig 1 Canine hypoglycaemia alarm (Candy): (top) alert mode; (bottom) activated

\section{Summary points \\ The reliable detection of hypoglycaemia has long been a major preoccupation for diabetic patients and researchers alike \\ Many previous approaches have been barking up the wrong tree}

We believe that we have identified a more promising tree

sweating) to measurements of subcutaneous tissue glucose concentrations by glucose sensors. ${ }^{45}$ As yet, however, none has proved sufficiently reliable or unobtrusive to be useful in clinical practice.

We describe here a novel alarm system that can detect hypoglycaemia before the patient notices any symptoms and that operates robustly in a uniquely, patient friendly fashion. The reports were volunteered spontaneously and independently by the patients.

\section{Case 1}

A 66 year old woman developed type 2 diabetes in 1971 and was transferred to insulin in 1979. She currently takes injections of soluble and isophane insulins (Humulin S and I; Lilly, Basingstoke) twice daily, totalling 38 units per day, and monitors her blood glucose at least once daily. She has no significant diabetic complications or other illness, and drinks very little alcohol. For the past two years she has experienced increasingly frequent hypoglycaemic episodes, with excessive sweating, generalised weakness, anxiety, and irritability. Most attacks occur in the evening and some occur at night. She retains generally good awareness of hypoglycaemic symptoms and can usually take quick corrective action.

Over the past year she has noticed unusual stereotyped behaviour displayed by Candy, her 9 year old mongrel bitch, which occurs only before hypoglycaemic episodes. Candy jumps up, runs out of the room, and hides under a chair in the hallway (fig 1), and re-emerges only when the patient has taken carbohydrate. Intriguingly, Candy acts in this way before the patient is aware of any hypoglycaemic

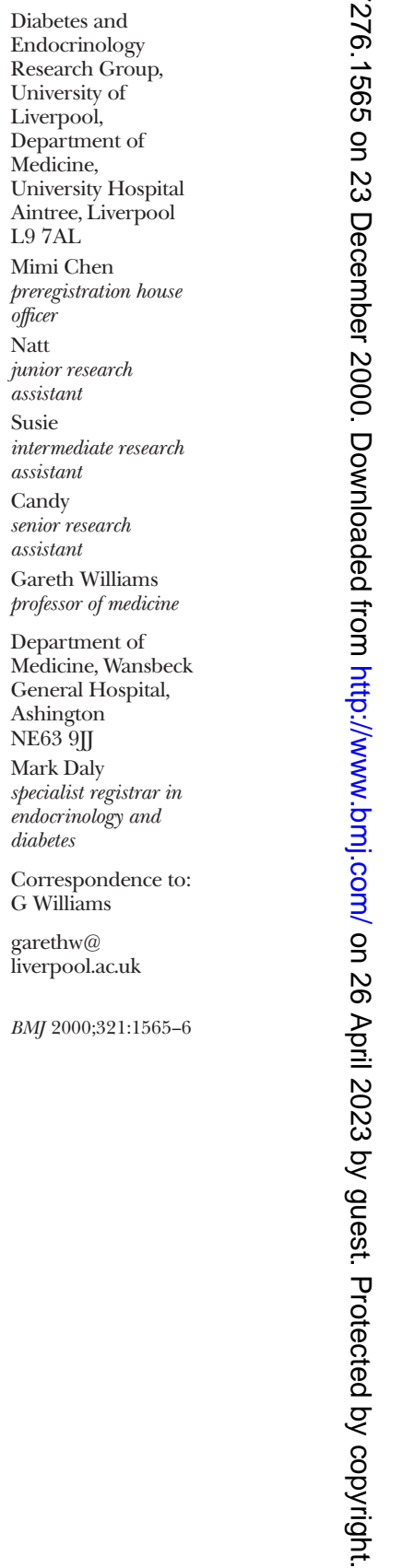


symptoms; blood glucose concentration during such episodes is around $1.5 \mathrm{mmol} / \mathrm{l}$.

\section{Case 2}

A 47 year old woman has required insulin since type 2 diabetes was diagnosed in 1995 . She has no diabetic complications, drinks hardly any alcohol, and takes Mixtard insulin (Novo Nordisk, Crawley) twice daily (total 28 units per day). She has up to two hypoglycaemic episodes each week, usually with good warning and characterised by sweating, malaise, and sometimes confusion. Episodes tend to occur during the afternoon and sometimes at night.

Within the past year her 7 year old mongrel bitch, Susie, has shown peculiar behaviour during the patient's hypoglycaemic attacks. At night she has been nudged awake by Susie and has then found herself to be hypoglycaemic. Susie goes back to sleep only after the patient has taken carbohydrate and her symptoms have settled; interestingly, her husband sleeps throughout. On other occasions, Susie has refused her favourite chocolate treats and has prevented the patient from leaving the house until she has taken food to correct hypoglycaemia. During most of these episodes the patient has initially been unaware that she is becoming hypoglycaemic, but blood glucose is around $2 \mathrm{mmol} / \mathrm{l}$.

\section{Case 3}

A 34 year old woman has had type 1 diabetes since 1970, complicated by retinopathy and nephropathy. She currently takes Humulin I and lispro insulin totalling 41 units per day, does not smoke, and drinks very little alcohol. On average, she has two hypoglycaemic episodes per week, with sweating and light headedness; she has reduced awareness of hypoglycaemia and does not wake up during nocturnal episodes.

Her 3 year old golden retriever, Natt, becomes very distressed whenever she is hypoglycaemic. During the day, he paces up and down and puts his head on her lap; during nocturnal episodes, he barks and scrabbles against the bedroom door. Natt only settles once her hypoglycaemia has been corrected. Blood glucose concentration on two such occasions was 1.6 and 1.9 $\mathrm{mmol} / \mathrm{l}$.

\section{Discussion}

Over a third of dogs living with diabetic people have been reported to show various behavioural changes during their owners' hypoglycaemic episodes. ${ }^{6}$ The

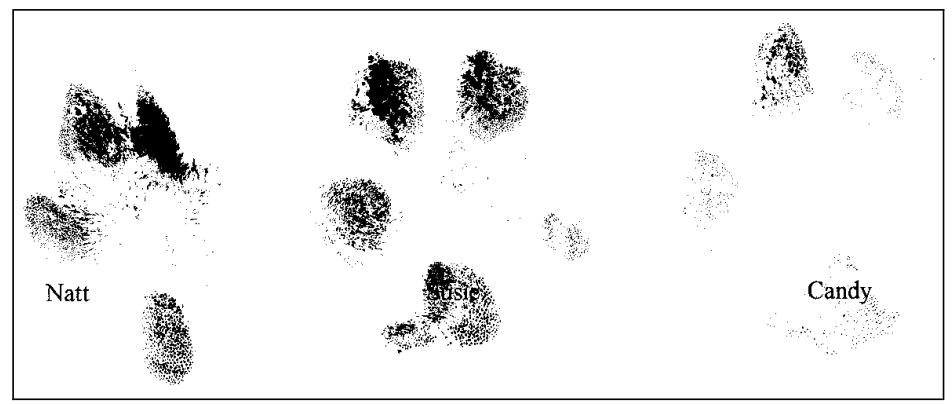

The issue of authorship is taken seriously by responsible journals three dogs reported here take canine glucose sensing to a new level of sophistication. All were clearly able to sense hypoglycaemia accurately under circumstances when the patients themselves were initially unaware of falling glucose levels. Formal calculations of sensitivity and specificity are not possible, but each dog showed her specific behaviours only when the patient had documented hypoglycaemia. Susie and Natt deserve special mention because they were able to detect nocturnal episodes in their owners and then undertook further corrective action by waking them to eat-thus going further than any available glucose sensor.

These two cases hint at hitherto unsuspected ways in which hypoglycaemia may be detected, even before symptoms are noticed by people whose hypoglycaemia awareness is mostly intact. The physiological basis is uncertain, but direct contact with the diabetic patient was not required in any of these cases. Possible clues include olfactory changes (possibly related to sweating), muscle tremor, or behavioural alterations such as the patient's failure to respond to her dog in her usual way. We are attracted by the notion of the "sixth sense" with which dogs are commonly credited, but acknowledge that this will need to be substantiated by further research.

As well as being useful in sniffing out drugs, explosives, and agricultural contraband, dogs have contributed generously to many aspects of medicine. Their achievements include guiding blind people, providing rapid-response paramedical assistance to people lost in the mountains, recognising fits in patients with epilepsy, ${ }^{7}$ and co-authoring papers in the $B M J{ }^{8}{ }^{8}$ Dogs are also beneficial to cardiovascular health-a particularly important issue in diabetic patients. Blood pressure is lowered by stroking dogs, ${ }^{9}$ and pets who drag their reluctant owners out for brisk 45-minute walkies are operating in accord with the WHO guidelines on physical exercise. $^{10}$

An extended healthcare role should now be considered for man's (and woman's) best friend. Research is urgently needed to determine whether dogs can be trained to recognise and react to early signs of hypoglycaemia. Hypoglycaemia alarm dogs could provide an important aid to patients with poor awareness of symptoms, particularly those prone to nocturnal episodes or who live alone.

Funding: None.

Competing interests: Bonio (N, S, and C).

1 Frier BJ. Hypoglycaemia in diabetes mellitus. In: PickupJ, Williams G, eds. Textbook of diabetes. 2nd ed. Oxford: Blackwell Science, 1997:40.1-40.23.

2 Weston PJ, Gill GV. Is undetected, autonomic dysfunction responsible for sudden death in type 1 diabetes mellitus? The 'dead in bed' syndrome revisited. Diabetic Med 1999;16:626-31

3 Pramming S, Thorsteinsson B, Bendtson I, Binder C. Symptomatic hypoglycaemia in 411 type 1 diabetic patients. Diabet Med 1991;8:217-22.

Pickup JC. Sensitive glucose sensing in diabetes. Lancet 2000;355:426-7.

5 Rebrin K, Steil GM, Van Antwerp WP, Mastrototaro JJ. Subcutaneous glucose predicts plasma glucose independent of insulin: implications for continuous monitoring. Am J Physio 1999;40:E561-71.

6 Lim K, Wilcox A, Fisher M, Burns-Cox CI. Type 1 diabetes and their pets. Diabetic Med 1992;9(suppl 2):S3-4.

7 Strong V. Seizure-alert dogs-fact or fiction? Seizure 1999 Feb;8:62-5.

8 Williams T, Kim, Williams G. Excessive impertinence or a missed diagnosis? BMJ 1995;311:1700-1.

9 Vormbrock JK, Grossberg JM. Cardiovascular effects of human-pet dog interactions. J Behav Med 1998;11:509-17.

10 WHO/International Federation of Sports Medicine Committee on Physical Activity for Health. Exercises for health. Geneva: WHO, 1997. 